\title{
Neue Hypothesen zur Bodenbildung auf Quartärablagerungen der Nordostschweiz
}

\begin{abstract}
:
Our investigations on soils of the northeastern part of Switzerland lead to the following conclusions:

1) Quaternary soils of different age can generally be separated by using the $\mathrm{pH}$-values of the $\mathrm{B}$-horizon. The validity of this method is restricted to topographic positions where there have been neither accumulation- nor erosion-processes.

2) There seem to be more textural inhomogeneities of the parent-material than previously supposed. Thus, soil development is often misinterpreted.

3) Special attention must be drawn to soil erosion on gravelly material. Otherwise, agricultural use of these soils can lead to irreparable damage within a short time because the weathering rate in gravels is low. In contrast, soil erosion in forested areas may lead to a slight amelioration as these soils are strongly acid and thus have a very low base-saturation.
\end{abstract}

\section{Key words}

soil erosion, soil acidity, soil age, quaternary soils

Dieser Artikel basiert auf Datenmaterial aus eigenen Untersuchungen, ergänzt durch Literaturhinweise und Resultate aus noch nicht veröffentlichten Diplomarbeiten (U. ZEHNDER. R. MEULI). Die Untersuchungen am Gubrist wurden durch den Schweiz. Nationalfonds und die Stiftung für wissenschaftliche Forschung an der Universität Zürich mitgetragen.

\section{Die typischen Böden der Nordostschweiz}

Gemäß dem ATLAS DER SCHWEIZ (1984) dominieren in dieser Region Braunerden, pseudovergleyte Braunerden und Parabraunerden, und zwar in Abhängigkeit einmal vom geologischen Ausgangsmaterial (Molasse, Moräne, Schotter) und zum andern grob klimatisch-zonal von den nach Norden abnehmenden Niederschlägen.

Eine zu hohe Priorität wird nach unseren Erfahrungen der neutralen Braunerde, die typisch für eine geringe Verwitterung ist, eingeräumt: FREY (1983) erwähnt, daß die neutrale Braunerde meist an topographische und lithologische Besonderheiten gebunden ist. Neutrale Braunerden oder sogar noch kalkhaltige Vorstufen (Kalkbraunerden) finden sich unter natürlichen Verhältnissen nur in erosionsanfälligen, steilen Lagen bzw. in jungen Flußalluvionen und kolluvialen Überschüttungen. In vielen Fällen aber täuschen die landwirtschaftlich genutzten und bis in den Neutralbereich gedüngten (ursprünglich sauren) Braunerden eine zu geringe Verwitterung vor (z. B. Profil US 36, Blatt Uster, FAP 1981).

Genetisch gesehen ist die reife Bodenbildung auf letztkaltzeitlichem Material ein mäßig bis stark versauerter Boden von Braunerde- oder Parabraunerdecharakter und dessen pseudovergleyte Varianten. Neutrale Böden stellen aus irgendwelchen Gründen immer Ausnahmen dar. Als Beispiel für eine solche «klassische» Parabraunerde sei die Lokalität "Alten» dargestellt (Fig. 1). Deutlich ist dabei die Tondifferenz zwischen dem E- und dem Bt-Horizont zu erkennen, nachgezeichnet auch durch das pedogene Eisen. Die Lokalität befindet sich im Zungenbekken des Schlierenäquivalents des Thurlappens, die Unterlage des Profils, also der C-Horizont, besteht aus Schotter. Fraglich ist allerdings - wie auch später noch diskutiert wird -, ob sich die Parabraunerde tatsächlich aus diesem Schotter oder nicht vielmehr in einem darüberliegenden Moränenschleier und Hangschuttmaterial entwickelt hat. Es erhebt sich also hier die Frage nach einer Zweischichtigkeit (allenfalls sogar einer Mehrschichtigkeit) des Bodenprofils, weshalb die Bezeichnung (II) eingefügt wurde. Die durchschnittliche Mächtigkeit solch typischer holozäner Bodenbildungen liegt zwischen 1 und $1,5 \mathrm{~m}$, doch können lokal erosiv oder akkumulativ bedingte Abweichungen nach unten oder oben beobachtet werden.

Peter Fitze, PD Dr., Geograph. Institut der Universität ZürichIrchel, Winterthurerstr. 190, 8057 Zürich 


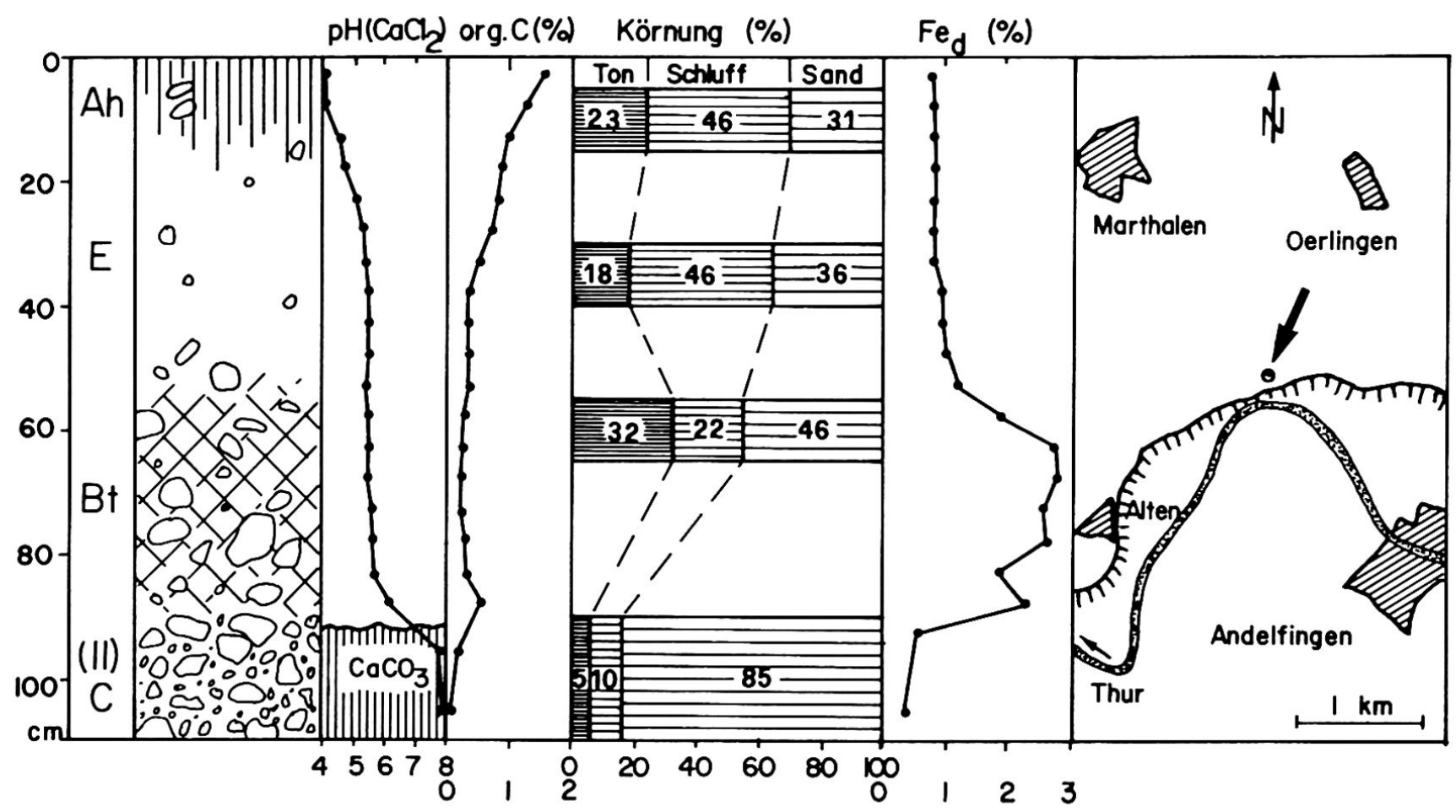

Fig. 1 Holozäne Parabraunerde, Lokalität "Alten"

Holocene luvisol, locality "Alten"

\section{Die Tiefenversauerung als Maß für das Bodenalter}

Ausgehend von dieser holozänen Parabraunerde ist es möglich, andersaltrige Bodenbildungen davon zu trennen. Damit ergibt sich auch für die Quartärmorphologie ein neues Gliederungskriterium, allerdings auf solche Reliefpositionen beschränkt, die weder als Gewinn- noch als Verlustlagen anzusprechen sind.

Bei den in Fig. 2 dargestellten pH-Tiefenfunktionen handelt es sich um einige ausgewählte Profile, die momentan noch keine gesicherten Schlüsse zulassen. Immerhin ist zu erkennen, daß die jüngsten Profile I-III die geringste Versauerung zeigen, nicht im Oberboden (hier scheint sich bereits nach relativ kurzer Bildungszeit ein kaum noch veränderbarer Reifezustand eingestellt zu haben), sondern in 0.5 bis $1 \mathrm{~m}$ Bodentiefe. Während Profil I als junges Hangschuttprofil (Kalkregosol) zu deuten ist, liegt Profil III auf der höchsten Verebnung des Gubrists (vgl. Lage in Fig. 5) und stellt den reifen Bodentyp dieser Gegend dar, eine saure Braunerde. Nicht grundsätzlich davon unterscheidet sich das Hangprofil II, das bei einer geringfügig stärkeren Versauerung des Unterbodens eine leichte Profilverkürzung aufweist, die auf Hangerosion zurückzuführen ist. Deutlich zeigt sich bei allen Profilen der
Zusammenhang zwischen Karbonatgehalt und $\mathrm{pH}$ Wert, am Gubrist ist oberhalb von pH 6,75 normalerweise Karbonat vorhanden (bei den eigenen $\mathrm{pH}$ Werten handelt es sich um $\mathrm{pH}\left[\mathrm{CaCl}_{2}\right]$-Werte). Aufgrund unserer Erfahrungen müssen wir also annehmen, daß es sich bei diesen Böden um Bildungen auf würmzeitlichem Material handelt und daß somit der ganze Gubrist während der letzten Kaltzeit von Eis bedeckt gewesen sein muß, was bislang nicht schlüssig nachzuweisen war.

Grundsätzlich andere Verhältnisse zeigen sich bei den beiden nahe beieinanderliegenden Profilen $\mathrm{V}$ und VI. Ihre Lage auf der als rißzeitlich gedeuteten Hochebene am "Chatzenstieg» SW von Glattfelden dürfte - was Materialgewinn oder -verlust betrifft als bilanzfrei eingestuft werden. Das dem Hochterrassenschotter aufliegende Feinmaterialpaket von rund $2 \mathrm{~m}$ Mächtigkeit dürfte dabei verschwemmter rißzeitlicher Löß sein. Die Entkarbonatisierungstiefe liegt aufgrund mehrerer Sondierbohrungen in 5 bis $6 \mathrm{~m}$ Tiefe und entspricht somit einer wesentlich länger andauernden Bodenbildung als am Gubrist. Es handelt sich hier um Pseudogleye, deren Initialisierung bereits im letzten Interglazial erfolgte und die sich bis heute weiterbilden konnten ohne nennenswerte Beeinträchtigung während der letzten Eiszeit, als an dieser Stelle ein Periglazialklima geherrscht hatte. 
Nochmals eine Stufe verstärkter Versauerung zeigt das dem Autor ebenfalls bekannte ProfilVII aus der Literatur (CONRADIN, 1982). In diesem (Deckenschotter) ist die Versauerung nochmals intensiver und umfaßt eine Tiefe von etwa $7 \mathrm{~m}$. Der gleichmäBige Verlauf der $\mathrm{pH}$-Kurve spricht gegen eine durch Akkumulation von verwittertem Material überhöhte Mächtigkeit. Ein ähnlich tiefgründig verwittertes Profil auf höherem Deckenschotter wird in den Erläuterungen zur Bodenkarte «Uster» (FAP, 1981) vom Stoffel in der Nähe von Hittnau beschrieben ( $\mathrm{pH}$-Wert in $240 \mathrm{~cm}$ Tiefe $=4,0$ ).

Das Lößprofil IV - ebenfalls der Literatur entnommen (ETH, 1981) - zeigt wahrscheinlich eine Zweischichtigkeit, indem stark verwitterter (Riß?-)Löß von 2 bis 2,5 m Mächtigkeit diskordant einem karbonhaltigen Löß aufliegt. Bei der oberen Lößlage handelt es sich also aufgrund der Profildaten um Sekundärlöß, womit eine Altereinstufung dieses Bodens sehr problematisch wird. Gerade dieses letzte Profil soll aufzeigen, daß eine Interpretation in vielen Fällen schwierig ist. Es sei auch nicht verschwiegen, daß noch Ungereimtheiten existieren. So zeigt beispielsweise das Profil von Steig auf der Irchelverebnung des höheren Deckenschotters (ETH, 1983) eine erstaunlich geringe Versauerung: So liegen die pH-Werte in 110 bis $120 \mathrm{~cm}$ Tiefe bei 5,7 und in 150 bis $160 \mathrm{~cm}$ bei 5,8 . Diese $\mathrm{pH}$-Werte decken sich allerdings überhaupt nicht mit den viel früher von RICHARD (1950) gemessenen Werten im Profil «Irchel», das sich nur rund $500 \mathrm{~m}$ vom obenerwähnten Profil «Steig» entfernt befindet. Richard konnte an seinem früheren Profil in 130 bis $140 \mathrm{~cm}$ Bodentiefe einen pH-Wert von 4,8 messen, was schon besser in unser Schema paßt.

Ein grundsätzliches Problem bei solchen Vergleichen stellt natürlich die $\mathrm{pH}$-Messung selbst dar, vor allem, ob die Messung in Wasser oder einer Elektrolytlösung vorgenommen wurde. So sind gerade im stark sauren Bereich Unterschiede bis zu einer $\mathrm{pH}$ Einheit zwischen den Messungen mit $\mathrm{H}_{2} \mathrm{O}$ oder $\mathrm{CaCl}_{2}$ (bzw. $\mathrm{KCl}$ ) möglich. Trotz dieser allseits bekannten Tatsache werden leider heute immer noch Werte publiziert, die keine Rückschlüsse auf die Methode zulassen und Vergleiche schwierig gestalten.

\section{Bodenerosion - Katastrophe oder Notwendigkeit?}

Im Zusammenhang mit Maßnahmen bei der Bodenbearbeitung und dem Bodenschutz wird die Bodenerosion - gemeint ist hier die exzessive Bodenerosion - immer wieder angesprochen. Ein typisches Beispiel einer durch Bodenerosion geprägten Situa-

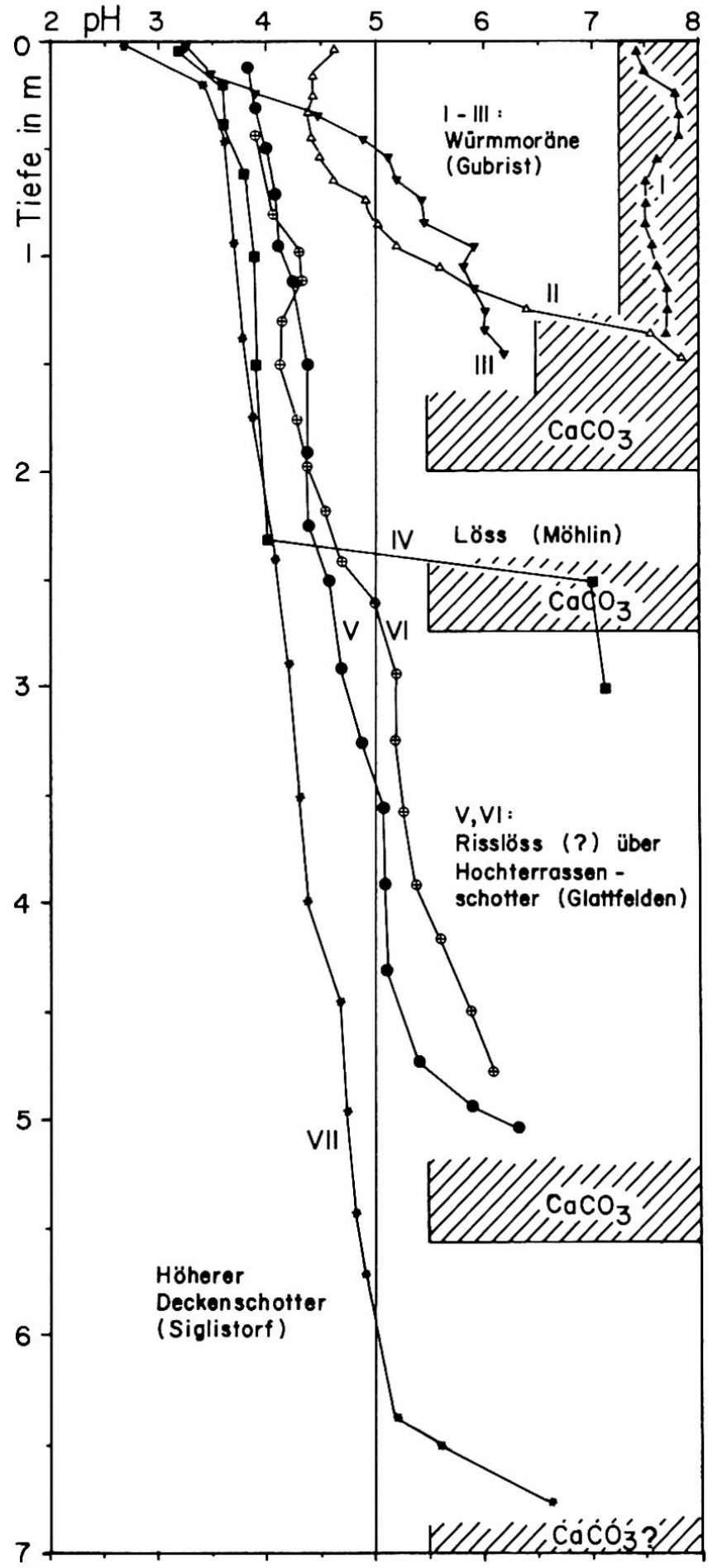

Fig. 2 pH-Tiefenfunktion der im Text beschriebenen Böden

$\mathrm{pH}$-depth-function of the soils described in this article 
tion gibt Fig. 3 wieder. Die Situation in der Kiesgrube von Wasterkingen ist insofern mit Fig. 1 vergleichbar, als sich auch hier eine "Deckschicht" über einem Schotter befindet. Die eigentliche Bodenbildung beschränkt sich auch hier im wesentlichen auf diese Deckschicht. Deutlich ist die durch Bodenerosion hervorgerufene Profilverkürzung am Mittelhang zu erkennen (hier sollte theoretisch die Bodenerosion auch am stärksten wirksam sein) sowie die abnormal große Mächtigkeit im Bereich des Unterhanges. Die Profilverkürzung am Mittelhang auf 40 bis $50 \mathrm{~cm}$ hat für die Landwirtschaft negative Folgen, sind hier doch die physiologische Gründigkeit sowie das Wasserspeichervermögen reduziert. Damit ist im Normalfall eine Ertragseinbuße verbunden, besonders in dem Fall, wo sich unter dem feinkörnigen Boden ein poröser Schotter befindet. Die Profilanalysen am Unterhang belegen die schon visuell sichtbare Gewinnsituation: Hohe $\mathrm{pH}$-Werte im Oberboden mit Karbonatspuren sowie einem im Vergleich zum Bv-Horizont sehr hohen Skelettgehalt des M-Horizontes. Einen solchen Bodentyp bezeichnen wir deshalb als eine neutrale Kolluvialbraunerde. Daß dies keine subjektive Interpretation ist, belegen im vorliegenden Profil Funde von Topfscherben sowie Holzkohlereste in rund $1 \mathrm{~m}$ Tiefe. Damit ist auch klar, daß es sich bei dieser Art von Bodenerosion um ein zeitlich junges Phänomen handelt und wahrscheinlich durch menschliche Eingriffe ausgelöst wurde. Wegen der Verlagerung von
Bodenmaterial kann eine kaltzeitliche Erscheinung mit Sicherheit ausgeschlossen werden, nicht von der Hand zu weisen ist allerdings, daß es sich bei dieser Deckschicht insgesamt um einen würmkaltzeitlichen Hangschutt (beispielsweise eine Solifluktionsdecke) handelt.

Damit drängt sich wieder wie bei Fig. 1 eine Zweischichtigkeit auf, eine Gliederung in eine obere, feinerdereiche Schicht (bei Fig. 160 , evtl. $90 \mathrm{~cm}$, bei Fig. 3 zwischen $M$ und $B v$ ), in der sich im wesentlichen die Bodenbildung abgespielt hat, und eine untere Schicht aus Grobmaterial ohne starke Verwitterungsmerkmale. Diese Erscheinung kennt man beispielsweise auch aus Deutschland, wo die jungtundrenzeitliche Deckschicht ebenfalls zu einer Zweischichtigkeit vieler Bodenprofile führt.

Meist handelt es sich bei der Bodenerosion aber um ein eher schleichendes Phänomen. Nach einer zusammenfassenden Studie der IUSA (1986) werden die weltweiten Bodenverluste von Ackerland jährlich auf etwa $21 \mathrm{t} /$ ha geschätzt, in der Schweiz aufgrund von Schwebstofferhebungen in den Gewässern auf etwa $1 \mathrm{t} / \mathrm{ha}$, was sicher einen Minimalwert darstellt. Dieser Wert entspricht ungefähr einem jährlichen Bodenabtrag von $0,1 \mathrm{~mm}$. Da die Bodenerosion als typischer Schwerkraftprozeß nicht prinzipiell vermieden werden kann, erhebt sich die Frage nach dem tolerierbaren Maß. Wenn man als grobe Schätzung für die holozäne Bodenbildung eine Zeitdauer von 10000 Jahren annimmt (mit ei-

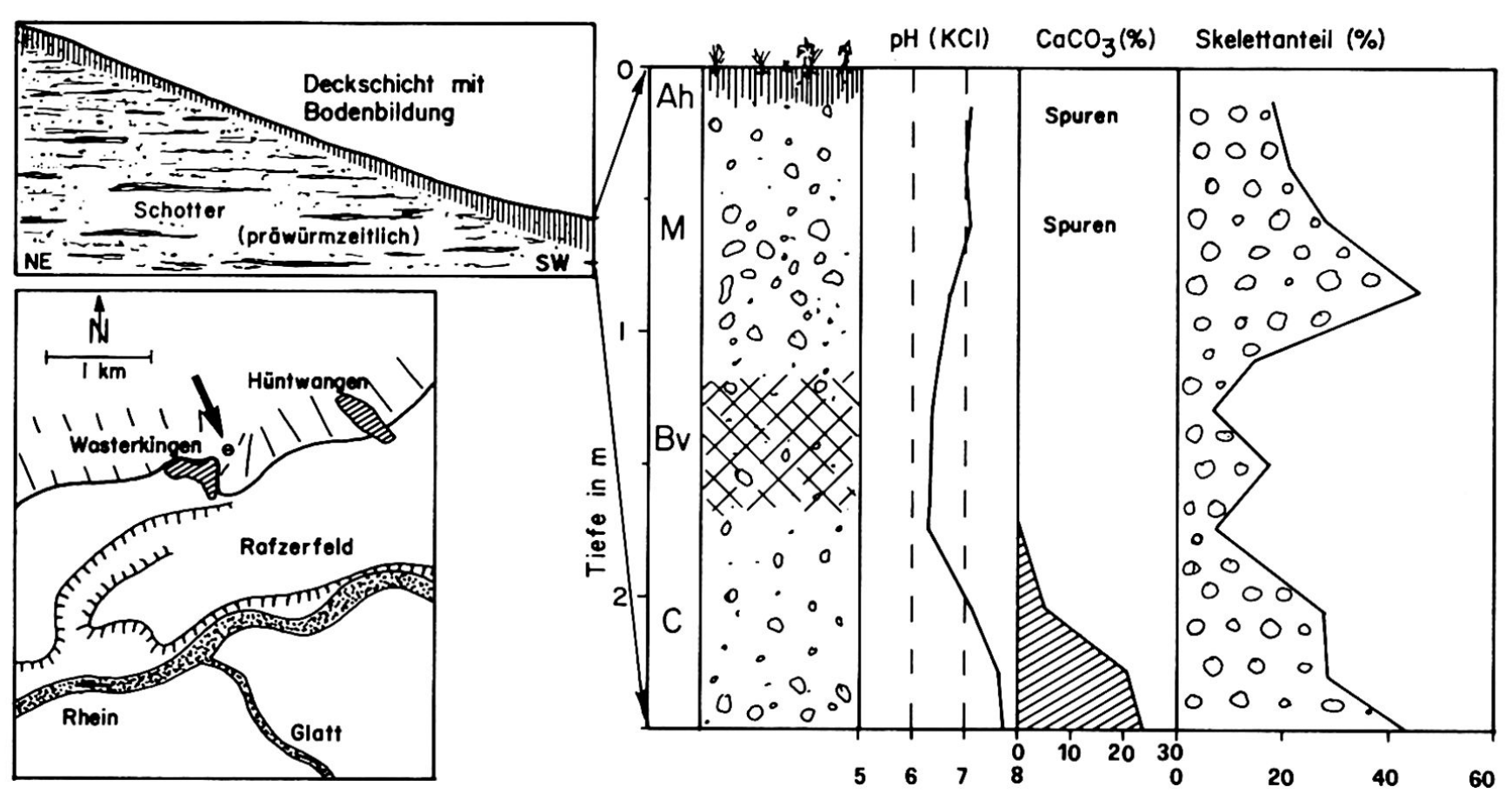

Fig. 3 Lokalität “Wasterkingen”, neutrale Kolluvialbraunerde

Colluvial Eutric cambisol, locality "Wasterkingen" 
nem Fehler von $50 \%$ ) bei einer Bodenmächtigkeit von $1 \mathrm{~m}$, läge die durchschnittliche Bodenbildung in der Größenordnung von $0,1 \mathrm{~mm} / \mathrm{J} a h r$ und könnte den erwähnten Bodenverlust gerade etwa wettmachen. Nun sind solche Zahlen aber aus verschiedenen Gründen nur als Größenordnung und nicht als beruhigende Feststellung zu betrachten:

- Die tatsächliche Zeitdauer für die Bildung des Holozänbodens ist nach wie vor wissenschaftlicher Streitpunkt. Zudem ist fraglich, ob die frühoder mittelholozänen Verhältnisse einfach auf die Gegenwart übertragen werden können.

- Die Schätzwerte für die Bildungsgeschwindigkeit gelten in erster Linie für die im Mittelland vorherrschenden Grundmoränen und andere feinmaterialreiche Substrate. Im Fall des Schotters muß mit einer verlangsamten Bodenbildung gerechnet werden, da das grobkörnige Material einerseits eine hohe Durchlässigkeit für das Sickerwasser besitzt und andererseits eine relativ kleine spezifische Oberfläche aufweist. Dies muß sich in einer weniger intensiven chemischen Verwitterung manifestieren. In solchen Fällen (der AufschlußWasterkingen belegt diese kritische Situation) kann sich ein Boden kaum mehr regenerieren.

- Bei landwirtschaftlicher Nutzung der Hangregionen ist in jedem Fall mit verstärktem Bodenabtrag zu rechnen. Vor allem brachliegende Flächen oder Kulturen, die eine längere Brachezeit mit sich ziehen, lassen die Bodenerosion rasch auf das 10- bis 20fache der erwähnten Größenordnung ansteigen (SCHAUB, 1985), was langfristig unweigerlich eine Fruchtbarkeitsminderung nach sich zieht. Aufgrund der schweizerischen Siedlungspolitik erwarten wir in Zukunft eher eine Verschärfung dieser Situation.

Einer solchen, in der Landwirtschaft gefürchteten, extremen Form des flächenhaften Bodenabtrags (wahrscheinlich besser als "Hangdenudation» zu bezeichnen) begegnen wir an den bewaldeten, mit Moräne bedeckten Molassehügeln des Mittellandes, beispielsweise am Gubrist nördlich des Limmattales. Hier haben an den steilen Hängen die Abtragungsprozesse unter Mitwirkung des Hangwassers (und möglicherweise auch des Menschen) dazu geführt, daß zum Teil neutrale bis karbonathaltige Molassesande oder das praktisch unverwitterte Moränenmaterial an der Oberfläche liegt (Fig. 4). In Form vom Hang abgesetzter Sackungsmassen und Wasseraustritten entlang der nun freiliegenden Molassemergel sind diese Phänomene deutlich zu erkennen. Das dazugehörende Histogramm der $\mathrm{pH}$ Werte der 170 Oberbodenproben widerspiegelt diese Situation (Fig. 5). Wohl sind erwartungsgemäß die versauerten Böden in der Mehrzahl, doch weisen rund $20 \%$ aller Proben dieser obersten Bodenschicht einen neutralen $\mathrm{pH}-$ Wert auf.
Trotz dieser starken Hangdynamik scheinen sich die Verhältnisse für die Waldvegetation nicht generell negativ auszuwirken. Einzig bei der Erosion in Kuppenlage, wo die mangelnde Wasserspeichermöglichkeit der nun anstehenden Molassesande zu einer lokalen Trockenstreßsituation führt (Dominanz von Föhre und Lärche), sind die Auswirkungen als eindeutige Verschlechterung der Situation zu deuten. Daneben gewinnt man aber aus den bisherigen Untersuchungen (FITZE, 1986), vor allem im Bereich der Nordabdachung, sogar den Eindruck einer leichten Verbesserung. So liegen beispielsweise die Humusgehalte in den neutralen Oberböden deut-
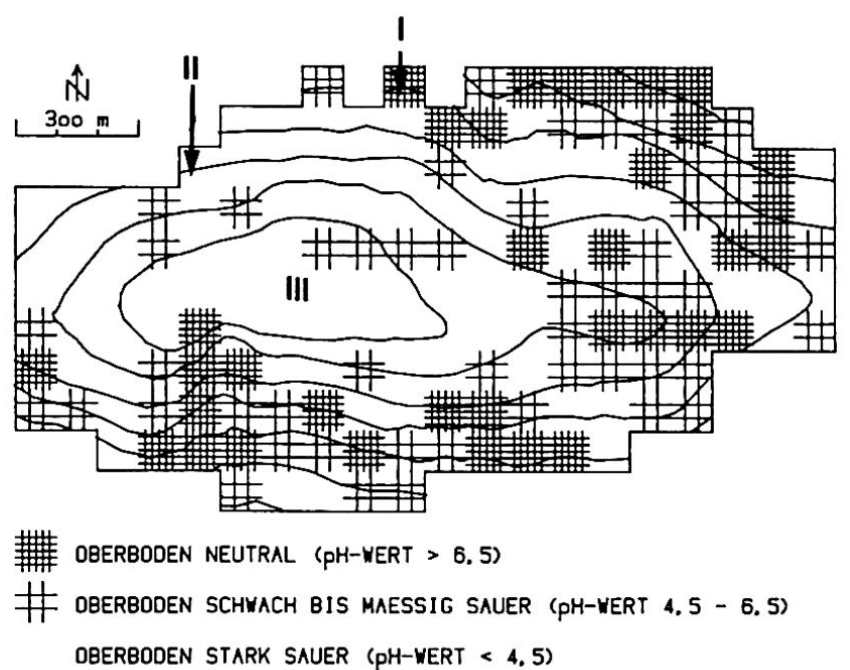

Fig. 4 pH-Verteilung des Oberbodens am Gubrist $\mathrm{pH}$-pattern of the A-horizon, Gubrist

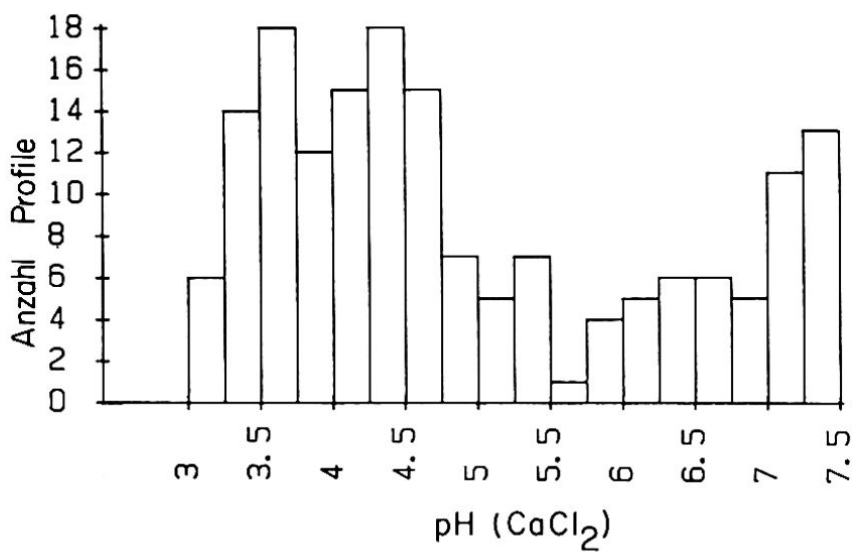

Fig. 5: Häufigkeitsverteilung der in Fig. 4 dargestellten $\mathrm{pH}$ Werte

Frequency of the $\mathrm{pH}$-values (A-horizons) from Fig. 4 
lich höher als bei den sauren (bei etwa gleichbleibenden $\mathrm{C} / \mathrm{N}$-Verhältnissen). Bei den Schwermetallen, die ja mehrheitlich erst im sauren Bereich löslich und damit pflanzenverfügbar sind, zeigen unsere Resultate, daß der neutrale Boden eine verstärkte Filterfunktion wahrnimmt, was sich schlußendlich auch auf das Trinkwasser auswirken wird. Wir vermuten, obwohl diesbezügliche Resultate zum Gesamtbodenhaushalt noch ausstehen, daß die im Hangbereich durch Erosion verminderte Wasserspeicherfähigkeit bzw. Gründigkeit durch (neutrales) Hangwasser ausgeglichen wird und $\mathrm{da} \beta$ die Nährstoffversorgung im Neutralbereich verbessert wird bei gleichzeitiger verstärkter Immobilisierung von Metallen, etwa dem toxischen Aluminium. Es muß allerdings unterstrichen werden, da $\beta$ diese letzten Feststellungen einer qualitativen Bodenverbesserung durch Bodenerosion sicher nicht für die Landwirtschaft gelten, wo eher mit einer Verschlechterung der Bodenqualität aufgrund von Erosionsprozessen gerechnet werden muß.

\section{Zusammenfassung}

Die Erkenntnisse aus unseren Bodenuntersuchungen in der Nordostschweiz lassen folgende Schlüsse zu:

1. Unterschiedlich alte Quartärböden lassen sich aufgrund ihres Versauerungsgrades voneinander trennen, allerdings nur, wenn es sich um bilanzfreie Standorte handelt.

2. Häufig zeichnet eine Bodenbildung die ursprünglich schon vorhandene Mehrschichtigkeit des Substrats einfach nach. Genetisch sind solche Böden nicht zweifelsfrei zu interpretieren.
3. Bei landwirtschaftlicher Nutzung muß der Bodenerosion vor allem dann Beachtung geschenkt werden, wenn das Substrat aus Schottern besteht. Unter Wald können Hangabtragungsprozesse oft auch eine leichte Verbesserung der Standortsituation bewirken.

\section{Literatur}

ATLAS DER SCHWEIZ (1984): Böden, Blatt 7a (2. Ausgabe). Bundesamt für Landestopographie, Bern.

CONRADIN, H. (1982): Bodenkundliche und Schwermineralogische Untersuchungen an Böden verschiedener Schotterfluren der Nordschweiz. Bodenk. Ges. der Schweiz, Bull. 6, 123-130.

ETH Zürich (1981, 1983): Physikalische Eigenschaften von Böden der Schweiz, Bände 2 und 3.

FAP (Eidg. Forschungsanstalt für den landw. Pflanzenbau) (1981): Resultate und Erläuterungen zur Bodenkarte Blatt 1092 Uster.

FITZE, P. (1986): Die räumliche Variation von Bodeneigenschaften unter Wald am Gubrist bei Zürich. Bodenk. Ges. der Schweiz, Bull. 10, 25-30.

FREY, E. (1983): Agrarpedologie. Geogr. Inst. Univ. Bern, $150 \mathrm{~S}$.

IUSA (Institut für Umwelt- und Systemanalyse) (1986): Erosion in der Schweiz: Ein Beitrag zur Standortbestimmung. Zürich, $53 \mathrm{~S}$.

RICHARD, F. (1950): Böden auf sedimentären Mischgesteinen im schweizerischen Mittelland. Mitt. EAFV, Bd. XXVI, H. 2, 750-836.

SCHAUB, D. (1985): Bodenerosion auf Ackerflächen im Möhliner Feld und Tafeljura. Mat. zur Physiogeographica, H. 8, 53-65. 